\title{
Visual Motion Responses of Neurons in the Caudal Area PE of Macaque Monkeys
}

\author{
Salvatore Squatrito, ${ }^{1}$ Milena Raffi, ${ }^{1}$ Maria Grazia Maioli, ${ }^{1}$ and Alexandra Battaglia-Mayer ${ }^{2}$ \\ 'University of Bologna, Department of Human and General Physiology, 40127 Bologna, Italy, and 2University of Rome "La \\ Sapienza", Department of Human Physiology and Pharmacology, 00185 Rome, Italy
}

Area PE of macaques has traditionally been considered a somatosensory association cortex. Recent studies, however, suggest that neurons of this and neighboring areas are involved in the visual control of movement, especially arm movement. We investigated the neuronal sensitivity to local visual stimuli of this region by recording neuronal activity in two behaving macaque monkeys trained in a simple visual fixation task. Recordings were performed from the dorsal surface of the caudal pole of the superior parietal lobule (SPL). Classical receptive fields (RFs) were mapped by using conventional static or moving luminous figures. We found that many neurons in this area were selectively activated by moving visual stimuli. Cell responses were tuned to the movement direction. RFs were usually large; their mean surface covered some $30 \times 30^{\circ}$ of the visual field. The fovea was often included into RF, in many cases it was along a RF side. The center of RFs was mainly located in the contralateral hemifield, although RFs having the center ipsilaterally sited were also found. No evident retinotopy was found. Visual neurons were especially concentrated in a region of the SPL likely corresponding to area PEc. These results suggest that the caudal part of area PE contains neuronal populations specifically signaling local visual motion, possibly encoding the direction of moving objects. These signals might well be suited for sensorimotor integration mechanisms aimed at motor acts.

Key words: extrastriate cortex; superior parietal lobule; area PEC; visual motion; visuomotor integration; macaque monkey
Monkey's area PE (Brodmann's area 5), classically known as a somatic sensorimotor association area, has been suggested to be involved in egocentric representation of reaching in the monkey (for review, see Lacquaniti et al., 1995; Kalaska, 1996). More specifically, the caudal pole of the superior parietal lobule (SPL), including anatomically and/or physiologically recognized areas V6A (Galletti et al., 1996), PEc (Pandya and Seltzer, 1982), and $7 \mathrm{~m}$ (Cavada and Goldman-Rakic, 1989) also labeled as PGm (Pandya and Seltzer, 1982), is currently described as a key region in a dorsal stream of signals linking somatosensory, as well as visual, inputs to motor commands for body movements, especially arm movements (Caminiti et al., 1996, 1998; Battaglia-Mayer et al., 2000). Although pure visual responses have been recorded from neurons of area V6A (Galletti et al., 1999), and retinal input also influences motor-related neurons in area PGm $/ 7 \mathrm{~m}$ (Ferraina et al., 1997), classical visual responses from PE neurons have not been reported until now. Nevertheless, sensorimotor coordination functions, like those occurring in the SPL, including area PE, require some neuronal coding of visual signals. Moreover, visual sensitivity of area PEc can be predicted by the fact that this area is a target of direct projections from visual area V6A (Shipp et al., 1998). Within the context of a basic study aimed at assessing functional visual properties of the SPL in monkeys, we have found that a substantial number of neurons in area PEc possess

Received Aug. 30, 2000; revised Nov. 27, 2000; accepted Dec. 7, 2000.

This work was supported by grants from the Department of University and Scientific Research of the Italian Government (Ministero dell' Universitá e della Ricerca Scientifica e Tecnologica). We are indebted with Mr. Andrea Meoni for his important technical contribution in the training of the animals and in the daily laboratory operations. We also thank Prof. Eugenio Riva Sanseverino for his helpful suggestions on theoretical and methodological aspects of this work.

Correspondence should be addressed to Dr. Salvatore Squatrito, Department of Human and General Physiology, University of Bologna, Piazza di Porta San Donato, 2, 40127 Bologna, Italy. E-mail: squatrito@biocfarm.unibo.it.

Copyright (C) 2001 Society for Neuroscience $\quad 0270-6474 / 01 / 210001-\bullet \$ 15.00 / 0$ visual properties, being especially sensitive, among other, to local visual motion, with direction selectivity and direction tuning.

\section{MATERIALS AND METHODS}

Two macaque monkeys (Macaca fascicularis) were trained to perform steady gaze fixation in a behavioral task in which they had to look, for several seconds each trial, at a small target displayed in the middle of a 19 inch computer monitor placed $28.5 \mathrm{~cm}$ from the eyes, ignoring conventional visual stimuli that were moved across the visual field.

Once the performance criterion was reached, during daily experimental sessions with the animal's head fixed, the activity of single cortical neurons was recorded extracellularly during the periods of gaze fixation, in the dark. Glass-coated elgiloy microelectrodes were driven through the intact dura by a remotely controlled stepping motor fixed on top of an 18 $\mathrm{mm}$ in-diameter metal chamber, previously cemented to the skull over the caudalmost portion of the dorsal SPL, along the midline. The chamber was centered at stereotaxic coordinates anteroposterior -14 for both animals, and, laterally, left 3.5 in one animal, zero in the other. Any surgery procedure was done under deep thiopental anesthesia $(15 \mathrm{mg} / \mathrm{kg}$, i.v.).

For every isolated unit, responsiveness to conventional visual stimuli was studied during steady fixation, by manually moving white solid bars around the screen, on foveal as well as parafoveal and peripheral locations. When visual responses were detected, the stimulus was adjusted, as to orientation, size, direction, and speed of movement, for the best

This article is published in The Journal of Neuroscience, Rapid Communications Section, which publishes brief, peerreviewed papers online, not in print. Rapid Communications are posted online approximately one month earlier than they would appear if printed. They are listed in the Table of Contents of the next open issue of JNeurosci. Cite this article as: JNeurosci, 2001, 21:RC130 (1-5). The publication date is the date of posting online at www.jneurosci.org.

http://www.jneurosci.org/cgi/content/full/4995 
response, and the borders and center of the receptive field (RF) were outlined. Trials were then begun with the stimulus flashing (with equal phases ON and OFF of $1 \mathrm{sec}$ ) on the RF, or moving across it at constant speed $(20$ or $30 \% \mathrm{sec})$ along eight standard directions, at $45^{\circ}$ angular interval. Usually eight replications were collected for each direction.

Eye position was continuously monitored, monocularly, with an optoelectronic system that uses the corneal reflection of an infrared light beam. The maximum fixation window was $5^{\circ}$.

At the end of all recording sessions, the animals were killed with an overdose of anesthetic. The brains were then removed, and the block of brain beneath the chamber was serially cut $(60-\mu \mathrm{m}$-thick). Every other section was stained with toluidine blue and examined for architectonic analysis. The cutting plane was defined so that the track of each electrode would lie in one or closely adjacent sections. Electrode tracks were then reconstructed with the help of the coordinates of each penetration and previously placed electrolytic microlesions.

\section{RESULTS}

The cortical region referred to in this paper is included in the dorsomedial aspect of SPL, limited to the gray matter of the superior parietal gyrus, and never extending for $>2 \mathrm{~mm}$ along the medial surface of the hemispheres. Laterally, this region never exceeded the middle of the dorsal part of the SPL and extended rostrally not $>5 \mathrm{~mm}$ from the lip of the parieto-occipital sulcus (POS). Caudally, the region did not extend to the anterior bank of POS. Figure 1 shows the details of the recording region and some exemplary electrode tracks. The architectonic features of this region, determined according to the criteria of Pandya and Seltzer (1982), were those of area PEc.

We made 147 successful electrode penetrations in three hemispheres (two left and one right). Approximately 211 single-unit sites were recorded. We collected 133 and studied them quantitatively. The other 78 were discarded from collection and quantitative analysis because the preliminary subjective test with visual stimuli gave no detectable sign of visual-related driving. Because very few cells were excited by the steady presentation of the stimulus within the receptive field, the data collection was made by testing the cells with a local visual stimulus made of a luminous bar (usually $2.6 \mathrm{~cd} / \mathrm{m}^{2}$ ), whose geometrical features had been selected during the preliminary subjective test, moving across the RF in several directions. Of the 133 neurons tested in such a way, 86 resulted in a significant difference $(p<0.05$ to two-tailed $t$ test) in mean firing rate during the stimulus presentation over the RF, in at least one direction, compared to the discharge during steady fixation without stimulation [spontaneous activity (SA)], implying that their activity was related to visual input. Among these, 83 units presented an excitatory response, and three were inhibited.

RF sizes were usually rather large, often covering a quadrant or an entire hemifield. Indeed, their limits were often not clear, because the discharge increased gradually above SA as the stimulus reached the RF center, and afterward the firing decreased slowly. For this reason RF shapes were often difficult to outline. For the sake of simplicity, we assumed square-shaped RFs whose side was measured by the response duration along the preferred direction, corrected for the mean latency of the area (this was assessed in a subset of units in which the stimulus started at approximately the middle of the RF). Measured this way, RF sides on the whole sample of excitatory visual-sensitive cells $(n=$ 83 ) ranged from 10 to $50^{\circ}$. Average side was $30^{\circ}\left(\operatorname{SD~} 11.10^{\circ}\right)$. Because of this large RF size, it was very common (41 of $83 ; 50 \%$ ) that the fovea was included within the RF. In other instances (15 of $83 ; 18 \%$ ) the fovea was along one of the RF sides, and for $32 \%$ (27 of 83) of the cases, the RF spared the fovea. Nevertheless, in almost all the cases the layout of the RF was asymmetrical with

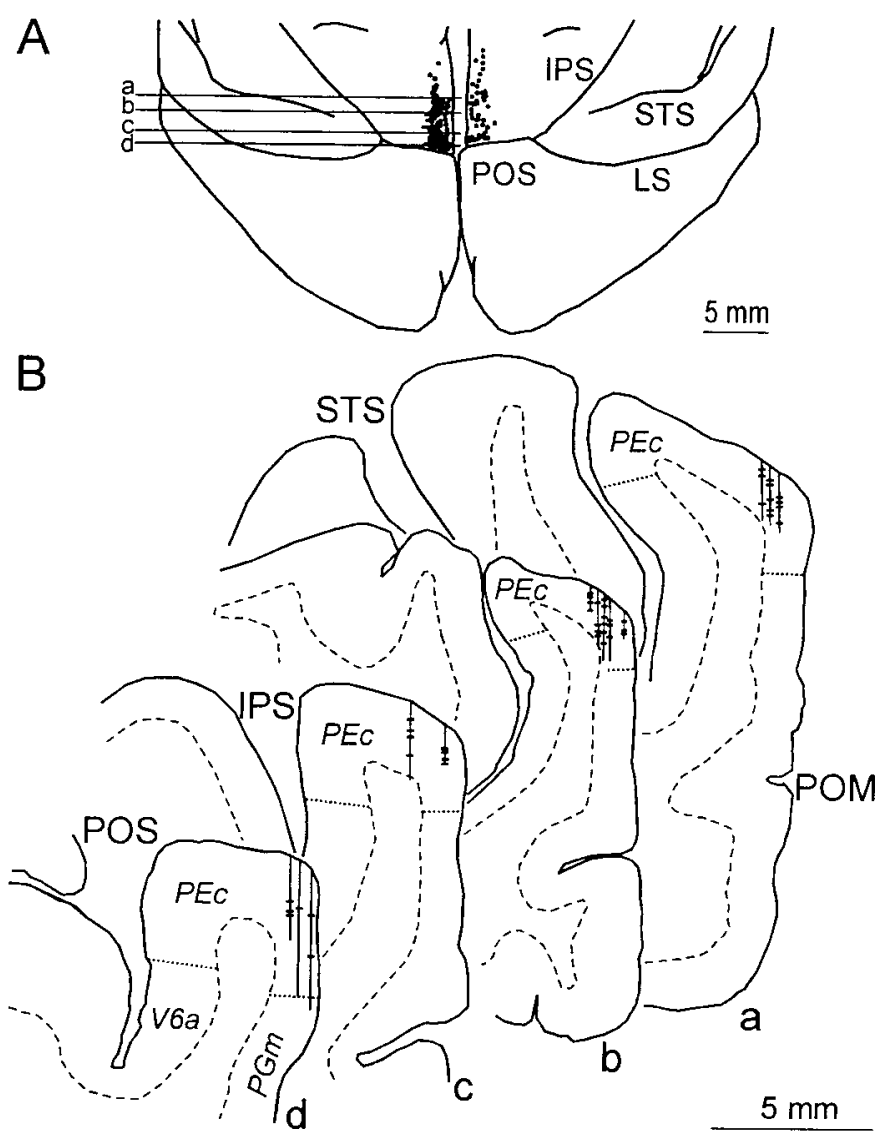

Figure 1. Recording region. A, Sketch of caudal half of macaque brain, showing the region of electrode penetrations within the superior parietal lobule. The dots indicate the entry points of electrodes perpendicular to the cortex. The drawing represents the brain of one of the two animals used in the experiments. On the left hemisphere the penetrations of the second monkey are superimposed (only the left side was used in this second animal). Horizontal lines indicate the levels of sections displayed in the bottom panel. $B$, Exemplary sections showing selected electrode tracks (vertical lines) with recording sites of visual cells (horizontal tacks). The plane of cut is perpendicular to the dorsal surface of the cortex at the anteroposterior level of POS. Electrode tracks were reported lying on the nearest serial section. Dotted lines mark the approximate limits of area $\mathrm{PEc}$, recognized on the basis of architectonics. IPS, Intraparietal sulcus; $L S$, lunate sulcus; $P O M$, medial parieto-occipital sulcus; $P O S$, parietooccipital sulcus; STS, superior temporal sulcus.

respect to the vertical or the horizontal meridian. Given this asymmetry of the RFs, we searched for laterality preferences. We grouped the RFs of each hemisphere in rightward or leftward skewed on the basis of the position of the RF center with respect to the vertical meridian. The great majority of RFs $(65 \% ; 54$ of 83 ) were contralaterally skewed (Fig. 2). Only 13\% (11 of 83) were ipsilaterally centered, whereas another $22 \%$ (18 of 83) had the center along the vertical meridian. As for the distribution with respect to the horizontal meridian, $18 \mathrm{RFs}$ were centered in the upper hemifield, 40 in the lower one, and 25 along the horizontal meridian.

Most often $(n=65)$ the RF structure was excitatory. Occasionally $(n=15)$ the RF consisted in side-by-side excitatory-inhibitory separate regions. In few cases $(n=2)$ visual neurons presented opponent vector organization features, as described by Motter and Mountcastle (1981) in the inferior parietal lobule.

Other parameters of visual stimuli, such as size, orientation, and luminous contrast, were tested during the preliminary assessment of response features of the neuron. Tested bar sizes ranged 
Left hemisphere

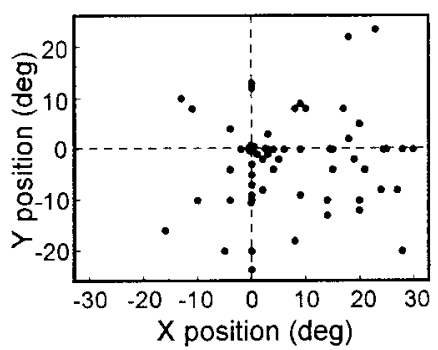

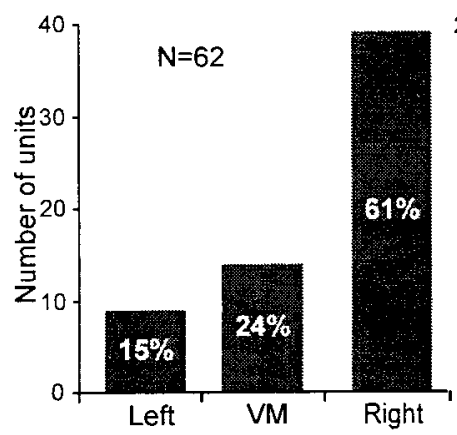

Right hemisphere
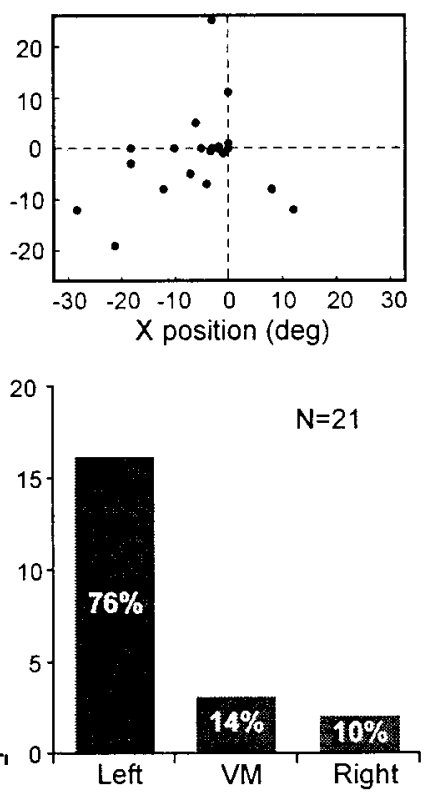

Figure 2. Receptive field spatial distribution. Top panels show the scatter of RF center positions of 83 units with excitatory responses to visual motion, subdivided by cerebral hemisphere. Bottom histograms report the frequency distribution of RF centers grouped by visual hemifield or vertical meridian $(V M)$.

from $0.1 \times 0.1^{\circ}$ of arc, up to $4 \times 1^{\circ}$ of arc, whereas bar orientations different from the one perpendicular to the preferred direction were also tried. No subjectively detectable discharge modulation was found in these tests. Thus, specific systematic stimulation series aimed at quantitatively studying these effects were not performed. As for the luminous contrast, occasionally it was changed by large steps (e.g., doubling or halving the figure luminous intensity). Inverted contrast stimulations (dark bars on white background) were also tried. In none of these tests evident discharge changes were detected. However, no study aimed at evaluating contrast thresholds was performed on these neurons.

We looked for retinotopic order of RFs along the surface of area PEc. For this we plotted, for each hemisphere, the RF center coordinates on the visual field against anatomical coordinates (anteroposterior and mediolateral axes) on a dorsal view of the SPL. No correlation was found between the two sorts of coordinates. Thus, although in each hemisphere the contralateral visual field is preferentially represented in PEc, visual RFs do not appear retinotopically ordered in this area.

The most evident feature of the visual neurons of the PEc was their selectivity for the direction of local visual motion. Usually the discharge rate during stimulus movement was best for few directions and equal to or below that of the SA for the opposite (null) directions. Figure 3 shows an example of this type of activation.

The analysis of directionality was performed on a subset of 73 units with all eight directions reliably tested. The other 10 units were discarded because only four to six collected directions were usable. Direction selectivity was first assessed by computing, for each unit, a one-way ANOVA across the eight directions. Almost all (71 of 73) tested neurons resulted with a $p<0.005$ to this test, implying they were direction-selective.

To assess the directional modulation of these neurons, we used

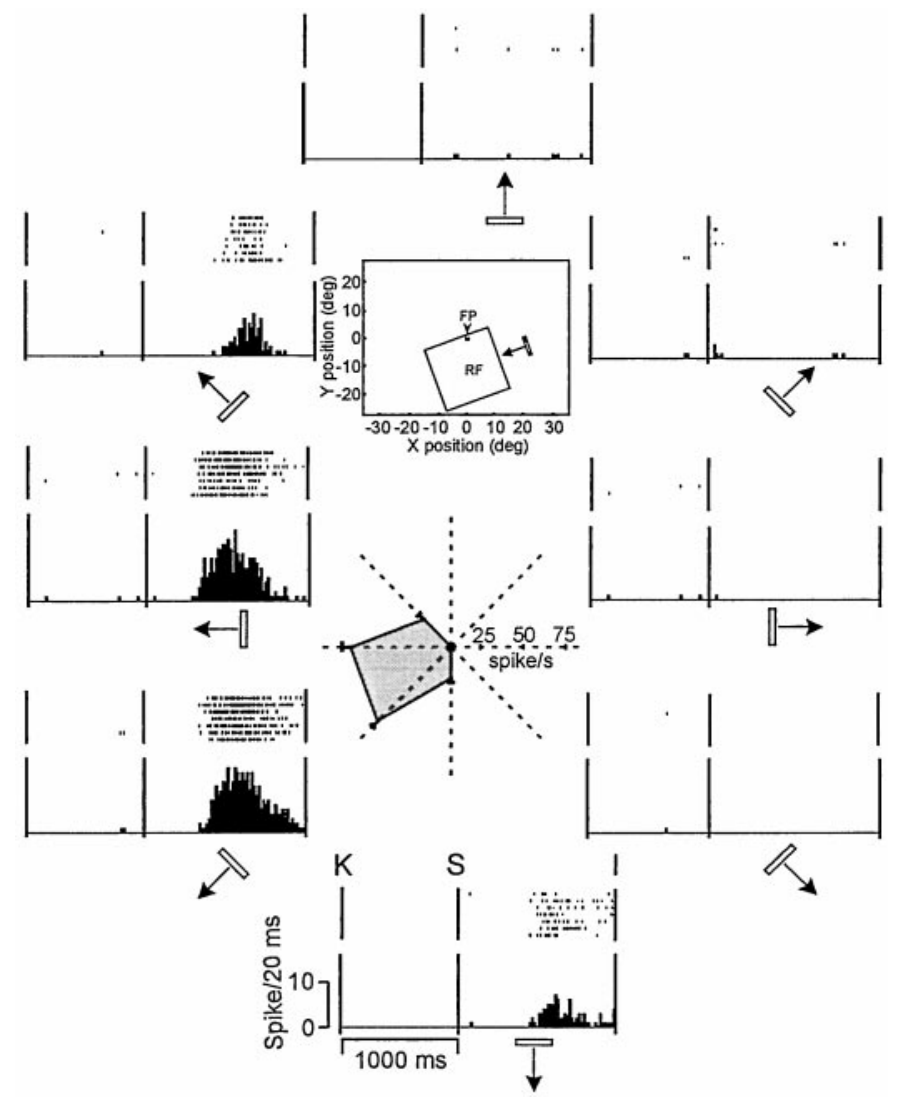

Figure 3. Direction tuning. Example of visual motion selectivity, and direction tuning, of a neuron from area PEc. The top sketch in the middle of the figure outlines the visual field covered by the screen in front of the animal. Scales of $x$ - and $y$-axes are in degrees of arc. FP is the fixation target, a square made of two $0.17^{\circ}$ wide vertical bars with a $0.17^{\circ}$ gap. RF outlines the visual receptive field of the neuron illustrated here. The arrowed bar represents a luminous figure moving across RF. Each peripheral figurine, made of paired raster dot and peristimulus time histogram $(P S T H)$, reports the cell discharge during both steady fixation without visual stimulation $(K-S)$ and stimulus movement across the RF $(S-I)$ of each of eight standard directions. Each direction is indicated by the arrowed bar below period S-I. Vertical scale on PSTHs represents the cumulative number of spikes in $20 \mathrm{msec}$ bins in seven trials. The polar plot in the bottom middle of the figure charts the mean firing rates (+SEM) of the eight responses to local visual motion with different directions.

the cosine tuning function with adjustable width described in Battaglia-Mayer et al. (2000). Neural activity, described as function of the direction $\alpha$ of stimulus motion, was fitted by:

$$
y(\alpha)= \begin{cases}A+K \cos \left(x^{*} S\right), & \text { if }|x * S|<\pi \\ A-K & \text { elsewhere. }\end{cases}
$$

In this model $x=\arccos [\cos (\alpha-\mathrm{C})] . A, K, C$, and $S$ are regression coefficients determined by a least-squares method. $C$ represents the "preferred direction", and $S$ is a parameter defining the angular interval in which $y(\alpha)$ is cosine-modulated. The results gave a high degree of directional tuning, because the coefficient of determination used to assess the goodness of fit of the cosine function was on average rather high $\left(R^{2}=0.90\right)$. Only four units (of 71) gave an $R^{2}<0.7$. The peak-to-peak amplitude of the tuning curves were assessed by a directionality index computed by the formula: 1 - (null response/preferred response), SA-subtracted (Maunsel and Van Essen, 1983). The great majority of direction-tuned neurons resulted in indexes $>0.5$, stressing 

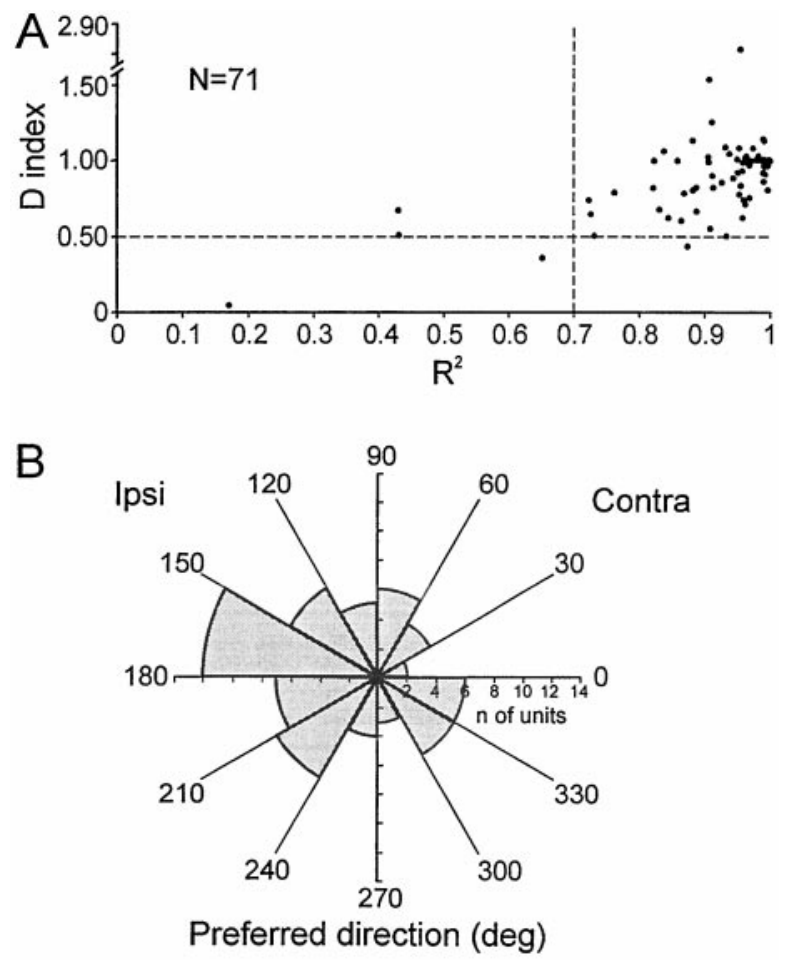

Figure 4. Directionality analysis. The analysis refers to a sample of 71 units, in which eight directions have been tested, and for which $p<0.005$ to ANOVA across the eight directions. $A$, Scatter plot of directionality index [ $d$ index $=1-$ (null response/preferred response), SA-subtracted] versus $R^{2}$ of cosine modulation analysis, as described in Battaglia-Mayer et al. (2000). The graph emphasizes how most of the direction-selective cells presented also a strong directionality, and this is related to the direction modulation. $B$, Rose diagram reporting the distribution of preferred directions, in $30^{\circ}$ bins, with respect to the side of the recording hemisphere. The preferred direction of each unit has been assessed by the cosine modulation analysis.

the strong directionality of these neurons. Figure $4 A$ shows a scatter plot of these indexes versus the $R^{2}$. Here it is evident how most of the visual responsive neurons with $R^{2}>0.7$ have also directional index $>0.5$, which means at least an increase by $100 \%$ of the response in the preferred direction with respect to the null direction.

The preferred directions resulting from the cosine analysis distributed around the $360^{\circ}$. No significant preferences for ipsilateral or contralateral directions, with regard to the hemisphere of recording were found (Fig. 4B). A slight preference for ipsilateral directions apparent from the rose diagram is not significant to the Rayleigh test of uniformity, performed on all the preferred directions. These findings suggest a vector coding of local visual motion direction by cell populations of caudal PE. Possible relationships between preferred direction of local motion within the RF and position of RF center with respect to the fixation point were searched by measuring the angle formed by the preferred direction and the axis joining the RF center to the fixation point. These angles distributed evenly around the $360^{\circ}$, suggesting a lack of any biases for centrifugal or centripetal directions.

\section{DISCUSSION}

The caudal pole of area PE, formerly reported as area 5b (Vogt and Vogt, 1919) or PEp (Von Bonin and Bailey, 1947) has been recognized as a uniform architectonic field, called area PEc, by
Pandya and Seltzer (1982). This area borders caudally on area V6A, at the anterior lip of POS, and medially on area PGm $/ 7 \mathrm{~m}$, within the mesial bank of the hemisphere. According to these architectonic criteria, our recording sites characterized by visual motion sensitivity could be assigned to area PEc.

It might be argued that the region from which we recorded partially overlapped area MDP described by Colby et al. (1988) and suggested as a possible relay of visual information between area PO and inferior parietal cortex. However, Colby's area MDP is entirely located on the medial surface of the hemisphere, and it appears to be coextensive rather with area $\mathrm{PGm} / 7 \mathrm{~m}$ than with PEc (Matelli et al., 1998; Shipp et al., 1998). Furthermore, the functional feature of a rapid habituation to visual stimulation, reported for neurons in MDP, does not agree with the visual responses reported in this work.

Present results do not agree with the statement by Galletti et al. (1999) that none of the cells in area PEc are responsive to visual stimuli. Indeed they use this criterion to define the dorsal limit of area V6A, a visual and oculomotor-related area lying on the anterior bank of POS. It seems unlikely that the recording region described in the present paper is a dorsal extension of area V6A, because our electrode penetrations, especially the most rich in visual neurons, are much more rostral with respect to the anterior lip of POS, where the dorsal limit of area V6A is commonly placed (Matelli et al., 1998; Shipp et al., 1998; Galletti et al., 1999), and they clearly fall within the dorsal aspect of the superior parietal gyrus (Fig. 1). Occasional recording sites located into the dorsal end of anterior bank of POS, possibly belonging to area V6A, were discarded from the database of the present work. It might be possible that the failure in finding visually sensitive neurons in PEc by Galletti et al. (1999) could be attributable to a partial electrode probing of this area in their experiments or to differences in the experimental conditions. A testable hypothesis is that visual neurons are not evenly distributed along the dorsal surface of the superior parietal gyrus, so that there might be some gap in the scattering of visual cells between area V6A and the region of PEc rich in visual cells. Our observations seem to suggest a detectable decrease in visual neurons in the most caudal penetrations. Nevertheless, the distribution of our penetrations was not uniform enough to significantly test this hypothesis. In the light of present results, the criterion of a lack of neurons with visual properties might not suffice to distinguish area PEc from V6A.

Despite the general role attributed to the caudal pole of the dorsal aspect of SPL, namely area PEc, as a high order somatosensory area (Matelli et al., 1998), there are no reports on functional properties of its neurons. The experiments reported in this paper demonstrate that a considerable analysis of local visual motion, especially with respect to the direction of stimulus movement, is performed by neurons of PEc. Thus, besides its possible involvement in other neuronal operations, this area should be included within the cortical network that processes visual motion.

As for the anatomical routes by which caudal $\mathrm{PE}$ can receive visual signals, there is no evidence, to our knowledge, of direct projections from areas V1-V3, or MT, to this area. Rather, visual signals may reach PEc trough areas V6A (Shipp et al., 1998), 7a, MIP, and MST (our unpublished results).

The dynamic features of PEc neurons reported above, such as the large RFs and the lack of a clear retinotopy, resemble more those of neurons in areas 7a and MST than those of cells in areas V1-V3 or MT. The functional similarity between PEc and MST, with regard to the processing of visual information, is confirmed 
also by the finding that PEc, like MSTd, may be a site of analysis of global visual motion such as optic flow, with selectivity for the position of the focus of expansion with respect to the fovea (Raffi et al., 1999). This sort of motion analysis is believed to be related to the visual perception of self-motion. Whether the local motion sensitivity demonstrated in the present study is functional to the optic flow selectivity or it is purposeful to the perception of object motion cannot be decided on the basis of actual results.

On the other hand, the role of the caudal pole of SPL, including areas V6A, PGm/7m, and possibly PEc in visual control of movement, particularly in visual reaching, is emphasized by recent physiological works (Caminiti et al., 1996, 1998; BattagliaMayer et al., 2000) (R. Caminiti, personal communication) and also predicted by the reciprocal association connections linking this region with dorsocaudal premotor cortex (Johnson et al., 1996; Wise et al., 1997; Matelli et al., 1998). These observations altogether suggest that the visual motion analysis, both local and global, performed in this region may serve for the combination of information about the movement of object or body in space with those concerning limb position for the composition of motor commands for reaching. Furthermore, these integration mechanisms might be crucial for the execution and control of motor acts such as object intercept and/or avoidance.

\section{REFERENCES}

Battaglia-Mayer A, Ferraina S, Mitsuda T, Marconi B, Genovesio A, Onorati P, Lacquaniti F, Caminiti R (2000) Early coding of reaching in the parieto-occipital cortex. J Neurophysiol 83:2374-2391.

Caminiti R, Ferraina S, Johnson PB (1996) The sources of visual information to the primate frontal lobe: a novel role for the superior parietal lobule (Review). Cereb Cortex 6:319-328.

Caminiti R, Ferraina S, Battaglia-Mayer A (1998) Visuomotor transformations: early cortical mechanisms of reaching (Review). Curr Opin Neurobiol 8:753-761.

Cavada C, Goldman-Rakic PS (1989) Posterior parietal cortex in rhesus monkey: I. Parcellation of areas based on distinctive limbic and sensory cortico-cortical connections. J Comp Neurol 287:393-421.

Colby CL, Gattas R, Olson CR, Gross CG (1988) Topographical orga- nization of cortical afferents to extrastriate visual area PO in the macaque: a dual tracer study. J Comp Neurol 269:392-413.

Ferraina S, Garasto MR, Battaglia-Mayer A, Ferraresi P, Johnson PB, Lacquaniti F, Caminiti R (1997) Visual control of hand reaching movement: activity in parietal area 7m. Eur J Neurosci 9:1090-1095.

Galletti C, Fattori P, Battaglini PP, Shipp S, Zeki S (1996) Functional demarcation of a border between areas V6 and V6A in the superior parietal gyrus of the macaque monkey. Eur J Neurosci 8:30-52.

Galletti C, Fattori P, Kutz D, Gamberini M (1999) Brain location and visual topography of cortical area V6A in the macaque monkey. Eur J Neurosci 11:575-582.

Johnson PB, Ferraina S, Bianchi L, Caminiti R (1996) Cortical networks for visual reaching: physiological and anatomical organization of frontal and parietal lobe arm regions. Cereb Cortex 6:102-119.

Kalaska JF (1996) Parietal cortex area 5 and visuomotor behavior. Can J Physiol Pharmacol 74:483-498.

Lacquaniti F, Guigon E, Bianchi L, Ferraina S, Caminiti R (1995) Representing spatial information for limb movement: the role of area 5 in the monkey. Cereb Cortex 5:391-409.

Matelli M, Govoni P, Galletti C, Kutz DF, Luppino G (1998) Superior area 6 afferents from the superior parietal lobule in the macaque monkey J Comp Neurol 402: 327-52.

Maunsel JHR, Van Essen DC (1983) Functional properties of neurons in middle temporal visual area of the macaque monkey. I. Selectivity for stimulus direction, speed, and orientation. J Neurophysiol 49:1127-1147.

Motter BC, Mountcastle VB (1981) The functional properties of the light-sensitive neurons of the posterior parietal cortex studied in waking monkeys: foveal sparing and opponent vector organization. J Neurosci 1:3-26.

Pandya DN, Seltzer B (1982) Intrinsic connections and architectonics of posterior parietal cortex in the rhesus monkey. J Comp Neurol 204:196-210.

Raffi M, Squatrito S, Maioli MG, Samolsky-Dekel B (1999) Visual responses of superior parietal lobule (SPL) neurons to radial optic flow. Soc Neurosci Abstr 25:672.

Shipp S, Blanton M, Zeki S (1998) A visuo-somatomotor pathway through superior parietal cortex in the macaque monkey: cortical connections of areas V6 and V6A. Eur J Neurosci 10:3171-3193.

Vogt C, Vogt O (1919) Ergebnisse unserer Hirnforschung. J Psychol Neurol 25:279-461.

Von Bonin G, Bailey P (1947) The Neocortex of Macaca mulatta. Urbana, IL: University of Illinois.

Wise SP, Boussaoud D, Johnson PB, Caminiti R (1997) Premotor and parietal cortex: corticocortical connectivity and combinatorial computations. Annu Rev Neurosci 20:25-42. 